\title{
GPPS-CH-2020-0017
}

\section{VALIDATION OF A SYNTHETIC-EDDY METHOD FOR MODELLING INCOMING WAKES IN SCALE-RESOLVING SIMULATIONS OF TURBOMACHINERY CASCADES}

\author{
Mark Zieße ${ }^{\star}$, Lars Wein \\ Junior Research Group Multiphysics of Turbulent Flows \\ Institute of Turbomachinery and Fluid Dynamics \\ Leibniz Universität Hannover \\ An der Universität 1 \\ 30823 Garbsen, Germany \\ Email: ziesse@tfd.uni-hannover.de
}

\author{
Christoph Müller-Schindewolffs, \\ Florian Herbst \\ MTU Aero Engines AG \\ Dachauer Str. 665 \\ 80995 München, Germany
}

\author{
Joerg R. Seume \\ Institute of Turbomachinery and Fluid Dynamics \\ Leibniz Universität Hannover \\ An der Universität 1 \\ 30823 Garbsen, Germany
}

\begin{abstract}
An enhanced Synthetic-Eddy Method for the modelling of free stream turbulence and incoming wakes for scale-resolving simulations of turbomachinery cascades is validated. It is shown that a previously presented method produces valid and physically plausible wakes representative of turbomachinery, and correctly reproduces their influence on the downstream cascade. The validation test case is an experimentally extensively investigated low-pressure turbine cascade with bars moving in pitch direction upstream of the cascade. Validation takes place in two steps: First, only the bars are considered. Then the profile including the moving bars is evaluated. In addition to the simulations with the directly modelled incoming wakes via the new method, the upstream wakes of the above two configurations are also directly resolved. The validation is proven by comparing the experimental values with the results of simulations with directly modelled wakes and the simulations with meshed moving bars.
\end{abstract}

\section{INTRODUCTION}

The wakes caused by the rotation of a turbomachine and the high turbulence levels outside the wake flow typical of turbomachines have a significant influence on the transition behaviour and thus also the losses of a turbomachine. The type of inflow therefore should be considered in the aerodynamic design of a turbomachine.

According to the current state of the technology, turbomachinery currently is aerodynamically designed using computeraided simulation methods known as Unsteady Reynolds Average Navier-Stokes Equations (URANS). A major disadvantage of these methods, however, is their modelling of turbulence. While current RANS methods have high prediction accuracies for steady inflows, Müller et al. (2015) could show which deficits current URANS methods have with regard to transient inflows, hence the current focus on this topic.

Due to their modelling, URANS procedures require extensive calibrations. Testing and calibration of URANS methods is currently carried out using experimentally investigated cascades. However, the generation of engine-like wakes upstream of the cascade to be investigated is difficult in the experiment. Either the Strouhal numbers (Stadtmüller et al., 2000) of current experimental investigations are clearly below those in a real engine, or a significant influence of the measurement setup cannot be excluded (Berrino et al., 2014).

Due to the steady decrease of the cost of computational resources, however, it is now possible to carry out so-called scaleresolving simulations (SRS) such as Large Eddy Simulation (LES) for technically relevant flows. In order to achieve signifi- 
Table 1 Geometric parameters of the validation test case

\begin{tabular}{lrrrrr}
\hline parameter & value & & parameter & value \\
\cline { 1 - 2 } \cline { 5 - 6 }$\beta_{\text {in }}$ & $127.7^{\circ}$ & & $l_{\mathrm{ax}}$ & $0.085 \mathrm{~m}$ \\
$b_{\text {wake }}$ & $0.040 \mathrm{~m}$ & & $\Delta x_{1}$ & $0.070 \mathrm{~m}$ \\
$b$ & $0.105 \mathrm{~m}$ & & $\Delta x_{2}$ & $0.060 \mathrm{~m}$ \\
$d_{\text {bar }}$ & $0.002 \mathrm{~m}$ & & $\Delta x_{3}$ & $0.030 \mathrm{~m}$ \\
\cline { 5 - 6 }$l$ & $0.100 \mathrm{~m}$ & & &
\end{tabular}

cantly better results with these SRS compared to the URANS methods, the simple application of the methods is insufficient. As mentioned before, an inflow similar to that encountered in the engine must also be considered. While in URANS procedures, the turbulent inflow can be directly specified due to the complete modeling of turbulence, in SRS, additional methods have to be used to generate the turbulent inflow.

For the generation of turbulent inflows in SRS different methods have been developed and successfully applied (Dhamankar et al., 2015). One method for generating inflow turbulence is the so-called Synthetic-Eddy Method (SEM). A turbulent inflow can generally be described as a superposition of eddy structures of different sizes, shapes and time scales. The most accessible approach for the generation of synthetic turbulence is thus the introduction of eddies into the computational domain whose fluctuations on average produce the desired turbulent inflow. In a previous paper (Zieße et al., 2019), the authors presented an improved SEM for the generation of free-stream turbulence and incoming wakes, called Wakes and Compressible Divergence-Free Synthetic-Eddy Method (W-DFSEM), in SRS of cascades. The basic idea is to replace preceding blade rows and their wake directly by means of W-DFSEM. There are three main advantages of using W-DFSEM:

1. An upstream blade does not need to be meshed and simulated (computational resource reduction by approx. 50\%)

2. no rotor-stator interface is required and

3. a higher flexibility with regards to different wake variations

The aim of this paper is to validate the W-DFSEM on the basis of the existing experimental data for low, non-engine-like wakes, in order to then be able to use the W-DFSEM to analyze new, still completely unknown profiles under the influence of engine-like boundary conditions, in particular wakes and freestream turbulence.

These simulations with LES and W-DFSEM can then serve as a further database for the improvement of current URANS methods in addition to the experimental data.

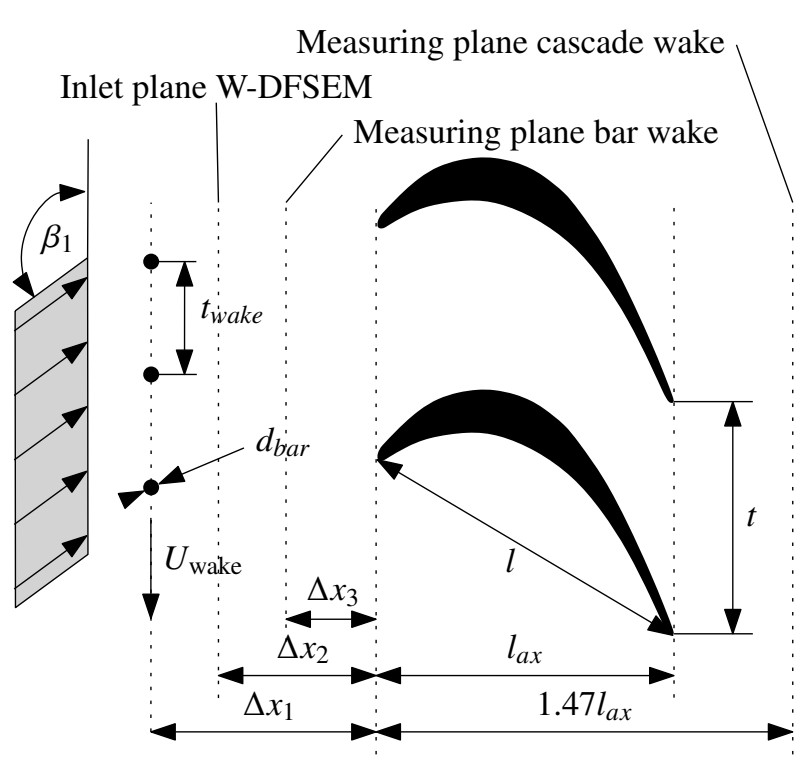

Figure 1 Definition of geometric parameters of the validation test case

\section{VALIDATION CONCEPT}

This paper mainly aims at validating the following two hypotheses:

1. With the W-DFSEM boundary condition previously presented in (Zieße et al., 2019), it is possible to generate physically plausible and sensible wakes as an inflow boundary condition of SRS

2. The influence of these wakes is correctly reproduced throughout the modelling chain.

The validation test case is the T106D low-pressure turbine cascade with upstream bars moving in pitch direction for wake generation. The $\mathrm{D}$ in the name of the cascade stands for a changed load of the profile $(b=0.105 \mathrm{~m})$ compared to the original T106A cascade $(b=0.08 \mathrm{~m})$. This test case was chosen because of the good data base. The test case was extensively investigated experimentally by Stadtmüller et al. (2000), Stadtmüller and Fottner (2001), and Hilgenfeld et al. (2002), as well as numerically by Blaim and Niehuis (2013). The selected operating point has an isentropic outflow Mach number $\mathrm{Ma}_{\text {out,is }}$ of 0.4 , isentropic Reynolds number $\mathrm{Re}_{\text {out,is }}$ of 200,000 and the wake movement velocity in pitch direction is $21.4 \mathrm{~m} \mathrm{~s}^{-1}$. The definition of all relevant geometric quantities of the validation are shown in Fig. 1. The corresponding values are listed in Tab. 2.

The entire validation concept can be divided into two main steps:

$1^{\text {st }}$ Step: Validation based on wakes of moving bars, without cascade

$2^{\text {nd }}$ Step: Validation of the influence of upstream bar wakes on a cascade

For both steps, the experiment is directly compared with the 
simulation with meshed and moving bars and with the simulation with incoming wakes generated directly with W-DFSEM. If this comparison shows reliable results for Step 1 and Step 2 , the W-DFSEM is validated for the generation of incoming wakes and the prediction of the influence of these wakes on a cascade. The simulations with meshed and moved bars make it possible to eliminate general methodical errors during the analysis of the artificially-generated wakes using W-DFSEM and can be used to quantify the accuracy of the wakes generated by W-DFSEM. In addition, this allows a direct comparison with regards to the required computational resources.

In principle, a comparison of the simulation with W-DFSEM and the experimental values for the second step would be sufficient to prove the validation. However, this would have the disadvantage that in case of significant deviations between simulation and experiment, it would not be possible to classify more precisely whether these deviations result directly from the wrongly generated wakes by the W-DFSEM or from general method errors (e.g. mesh resolution). This uncertainty can be excluded by the first step, i.e. using the test case with only bars but without the downstream cascade. Due to the measurement setup and the instrumentation used, it was only possible to measure the bar wakes with non-installed blades, and due to the influence of the potential field of the downstream cascade, a direct comparison of the wake measured without installed blades with the wake generated by W-DFSEM with installed blades is not a consistent comparison.

In addition to the simulations with moving wakes, simulations of non-moving bar and only cascade were also initially performed to evaluate the mesh error. The simulations of the non-moving bar are also used to determine the input parameters of W-DFSEM. For this purpose, the wake is extracted at the corresponding position behind the non-moving bar (see Fig. 1). For a multiple integer ratio of $t / t_{\text {wake }}$ the pitch of the bar was reduced to $0.035 \mathrm{~m}$. The domain size in span direction corresponds to $0.3 l$ for all simulations and the inlet of the simulations with meshed bars is $0.04 \mathrm{~m}$ upstream of the bars. The inlet of the simulations with W-DFSEM is $\Delta x_{2}$ upstream of the cascade. The outlet is located $\Delta x_{3}$ upstream of the cascade for the simulations with bar wake only and for the simulations including cascade $0.05 \mathrm{~m}$ downstream of the cascade.

The simulation of the cascade without incoming wake also served to determine the inflow angle. Due to the measuring setup for the generation of the moving bar wakes, deviations in the inflow angle already occur without installed bars. For the simulations carried out, a good agreement with the experiment was achieved with $\beta_{1}=133.2^{\circ}$. For further details please refer to (Stadtmüller, 2001).

\section{MODELLING DESCRIPTION}

The modeling of the computational domain itself is the same for all test cases. A finite volume method of second-order in space and time is used. The LES approach is used to reproduce the turbulence. The modeling of the smaller, isotropic structures necessary for this approach is done by the wall adapting local eddy-viscosity (WALE) subgrid-scale model (Franck and Ducros, 1999). The filter width is the mesh itself or a representative local cell size: the third root of the volume of the respective cell. The spatial discretization is done with the so-called LUST scheme. The LUST scheme is a combination of $25 \%$ second order upwind and $75 \%$ central differences schemes. The temporal discretization is done by means of the implicit second order backward schemata. For all simulations, it was ensured that the CFL number never exceeds 0.4.

The resulting equation system is solved using the solver rhoPimpleFoam for the test cases without moving mesh and for the test cases with moving mesh using rhoPimpleDyMFoam from the OpenFOAM software package. The DyM extension stands for dynamic mesh motion. The moving cells for the solution are taken into consideration by correcting the predominant fluxes in the affected cells. This correction is done for the simple case of continuous uniformly moving cells by replacing the fluid velocities $U$ in all convection terms with a relative velocity $U-U_{\text {wake. }}$ Then the same procedure for solving the equation system follows as for the solver rhoPimpleFoam. For more details, see (Jasak, 2009) and (Ferziger and Peric, 2001). Both solvers are based on an incompressible solver, which has been extended with a density correction.

For all test cases, the outer walls of the calculation domain are assumed to be periodic in pitch and span direction. In the case of adiabatic internal walls, like the walls of the bars and the blade, the boundary conditions of all the variables required for determination correspond to a Neumann boundary condition, except for velocity. The velocity is specified directly on the wall and thus corresponds to a Dirichlet boundary condition. For non-moving walls, the velocity at the wall is set to zero and for the moving bars the velocity at the wall corresponds to $U_{\text {wake }}$. As outlet boundary condition, the wave permeable waveTransmissive based on (Poinsot and Lelef, 1992) was used. This was also used at the inlet, except in the simulations with W-DFSEM where the velocity is specified by W-DFSEM.

\section{W-DFSEM}

The W-DFSEM is based on the Synthethic-Eddy Method by Jarrin et al. (2009). This in turn was extended by the property of divergence-freedom by Poletto et al. (2013) to the Divergence-Free Synthetic-Eddy Method (DFSEM). The SEM and DFSEM were primarily developed for flows close to walls. Müller-Schindewolffs and Herbst (2018) then optimized and recalibrated the DFSEM for turbomachinery in order, for example, to also consider periodic boundaries. In a further step, this Incompressible-DFSEM was coupled with the previously introduced waveTransmissive boundary condition to the Compressible-DFSEM. The C-DFSEM, on the other hand, was then extended by the specification of moving incoming wakes by Zieße et al. (2019) to the W-DFSEM and successfully verified.

The essential characteristics of moving wakes are a momentum 
deficit, increased turbulence and the unsteadiness itself. Inside the W-DFSEM the momentum deficit is set by specifying a velocity deficit based on the density function of the Gaussian distribution. This velocity deficit is clearly defined by a parameter for the wake width $\omega$, the wake depth $\sigma$, the velocity in free stream $U_{F S}$ and the number of wakes $N$. A 2D field of all Reynolds stresses of a non-moving wake is given for the reproduction of the increased turbulence. This 2D field was taken $\Delta x_{2}$ upstream of the cascade from the simulation of the nonmoving bar at midspan and the corresponding values for the velocity deficit are $\sigma=2.40, \omega=-0.23$ and $U_{\mathrm{FS}}=97.98 \mathrm{~m} \mathrm{~s}^{-1}$. For the unsteadiness itself, only the movement velocity of the wake in pitch direction $U_{\text {wake }}$ must be specified. The velocity deficit and the synthetic eddies are convected in pitch direction according to this velocity. In combination with these input parameters, the W-DFSEM allows to specify incoming wakes upstream in a SRS of a cascade. A brief explanation of the underlying equations of W-DFSEM is given in the appendix. For detailed description of the W-DFSEM please refer to (Zieße et al., 2019).

\section{RESULTS}

All experimental data shown are taken from (Stadtmüller, 2001). The absolute accuracy of the experimental results is $22 \mathrm{~Pa}$ for each pressure tapping of the profile pressure distributions and $6 \mathrm{~Pa}$ for the total pressure for each traverse position in the wake. The associated error bars are within the ranges of the symbol sizes, which is why they are not shown.

The ensemble averaging is based on the work of Lakshminarayana and Poncet (1974). Unless otherwise indicated, the number of averaged ensembles of the ensemble averaging is $M=25$ for the simulations with moving wakes and $M=300$ for the experimental results. The number of averaged ensembles in the experiment is thus 12 higher than in the simulations, which must be taken into account when interpreting the results (see Fig. 3). This lower number of ensembles in the simulations results from limited computational resources, but was chosen according to comparable numerical investigations (Michelassi et al., 2003; Michelassi et al., 2015; Koschichow et al., 2014). The number of samples of the temporal averaging for the simulations is about 700,000.

\section{Non-moving Bar}

The grid parameters of the grid study of the bar are listed in Tab. 2. $y_{\text {wall }}$ stands for the wall distance of the first cell layer in wall normal direction, $\Delta_{i}$ for a mean cell width far away from the wall, $R F$ for a refinement factor of the grids among themselves and $N$ for the total number of cells. Furthermore, the non-dimensional cell sizes tangential to wall $\Delta x^{+}$, normal to the wall $\Delta y^{+}$and in spanwise direction $\Delta z^{+}$are shown. The results of the grid study for the wake of the bar are shown in Fig. 2. In addition, an analytical velocity deficit based on the work of (Tennekes et al., 1972) is shown. For both grids $\mathrm{G}_{3}$ and
Table 2 Grid parameters of the 4 grids used for the grid refinement study of the bar.

\begin{tabular}{lcccc}
\hline para- & \multicolumn{4}{c}{ Grid } \\
meter & $\mathrm{G}_{1}$ & $\mathrm{G}_{2}$ & $\mathrm{G}_{3}$ & $\mathrm{G}_{4}$ \\
\hline$y_{\text {wall }}$ & $14.9 \mathrm{e}-6 \mathrm{~m}$ & $9.93 \mathrm{e}-6 \mathrm{~m}$ & $7.45 \mathrm{e}-6 \mathrm{~m}$ & $5.96 \mathrm{e}-6 \mathrm{~m}$ \\
$\Delta_{i}$ & $10 \mathrm{e}-4 \mathrm{~m}$ & $6.66 \mathrm{e}-4 \mathrm{~m}$ & $5 \mathrm{e}-4 \mathrm{~m}$ & $4 \mathrm{e}-4 \mathrm{~m}$ \\
$R F$ & 1.00 & 1.5 & 2.0 & 2.5 \\
$N$ & 149,520 & 519,345 & $1,221,960$ & $2,334,600$ \\
$\Delta x^{+}$ & 29 & 20 & 16 & 13 \\
$\Delta y^{+}$ & 2,1 & 1,6 & 1,3 & 1,0 \\
$\Delta z^{+}$ & 145 & 111 & 88 & 72 \\
\hline
\end{tabular}

$\mathrm{G}_{4}$ a good agreement with the analytical velocity deficit can be observed. Also the turbulent kinetic energy (TKE) distribution of these two grids largely matches. The grid $\mathrm{G}_{3}$ was therefore used for all further calculations, since it represents a good compromise between grid resolution and accuracy. In addition, this grid resolution corresponds to the simulations with cascade.

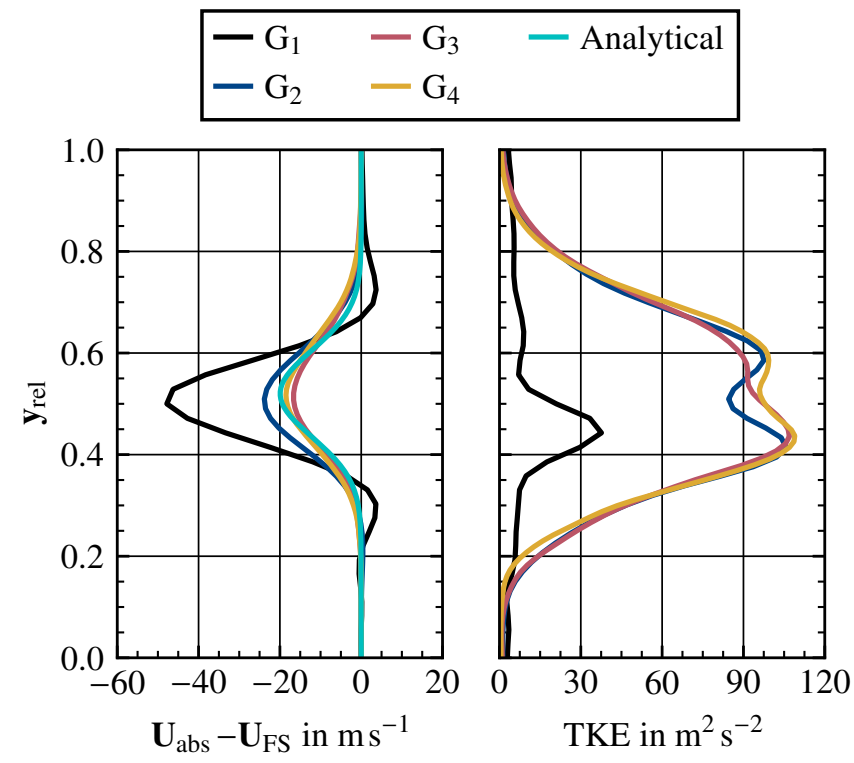

Figure 2 Velocity deficit (left) and TKE (right) in the wake at midspan of the grid study of the non-moving bar. 


\section{Moving Bar}

In Fig. 3, the results of the simulation with meshed and moving bar and the simulation of the moving bar wake directly modelled by W-DFSEM are compared with the results of the experiment. Additionally, the influence of the ensemble number of the ensemble-average on the results is visualised. All results shown were additionally shifted and averaged over the different phases. The wake width of the velocity deficit of the simulation with meshed bar agrees well with the experiment. The wake depth $\left(U_{\mathrm{FS}}-U_{\mathrm{abs}}\right) / U_{\mathrm{FS}}$ is slightly too high, which is due to the different number of averaged ensembles between experiment and simulation. The wake depth of the W-DFSEM agrees well with the experiment. However, the wake width of W-DFSEM is slightly too large, but could be further reduced by fine-tuning $\sigma, \omega$, and $U_{F S}$. A comparison of the TKE between moving bar, W-DFSEM and experiment shows a good agreement regarding the wake width. Differences between the simulations and the experiment can be observed in the height of the TKE peak. These differences can also be explained by the different number of ensembles.

In conclusion, there is a good agreement between the simulation of the moving bar, the simulation of W-DFSEM, and the experiment. The W-DFSEM thus correctly reproduces the moving bar wake and the first step of the validation concept is thus fulfilled.

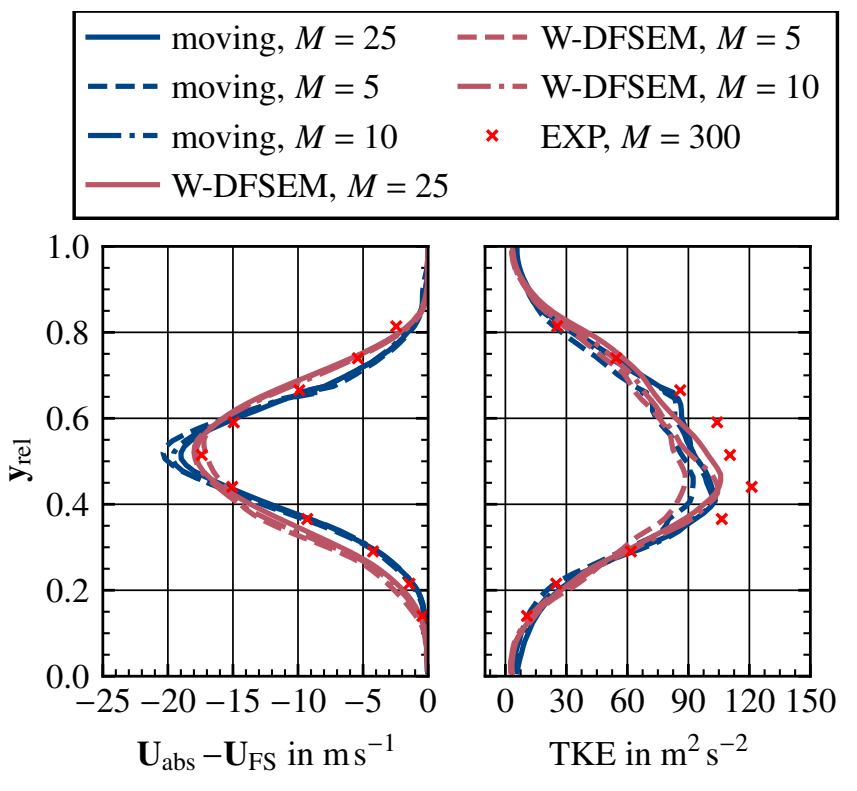

Figure 3 Comparison of the ensemble-averaged velocity deficit (left) and the TKE (right) in the wake at midspan $0.03 \mathrm{~m}$ upstream of the cascade for the simulation with meshed and moving bar and with bar wake modelled by W-DFSEM.
Table 3 Grid parameters of the 3 grids used for the grid refinement study of the cascade.

\begin{tabular}{cccc}
\hline $\begin{array}{c}\text { para- } \\
\text { meter }\end{array}$ & $\mathrm{G}_{1}$ & $\mathrm{G}_{2}$ & $\mathrm{G}_{3}$ \\
\hline$y_{\text {wall }}$ & $2.48 \mathrm{e}-5 \mathrm{~m}$ & $1.65 \mathrm{e}-5 \mathrm{~m}$ & $1.23 \mathrm{e}-5 \mathrm{~m}$ \\
$\Delta_{i}$ & $10 \mathrm{e}-4 \mathrm{~m}$ & $6.66 \mathrm{e}-4 \mathrm{~m}$ & $5 \mathrm{e}-4 \mathrm{~m}$ \\
$R F$ & 1.0 & 1.5 & 2.0 \\
$N$ & $2,569,020$ & $8,900,310$ & $21,039,449$ \\
$\Delta x^{+}$ & 31 & 18 & 12 \\
$\Delta y^{+}$ & 1,4 & 0,9 & 0,7 \\
$\Delta z^{+}$ & 58 & 39 & 28 \\
\hline
\end{tabular}

\section{Cascade}

The grid parameters of the grid study for the cascade are given in Tab. 3 and the corresponding results in Fig. 4. When the isentropic Mach number distribution is considered, a good agreement of all three grids can be observed, except for the front suction side area and the area of the separation bubble. In these areas the resolution of the grid $\mathrm{G}_{1}$ is not enough. This untypical mesh dependency in the area of the suction-side leading edge results from a small separation bubble in this area, graphically shown in Fig. 5. Between the two grids $\mathrm{G}_{2}$ and $\mathrm{G}_{3}$ no significant differences can be observed for the Mach number distribution and also for the suction-side wall shear stress no differences can be found between the two grids. When considering the total pressure loss in the wake, however, differences can also be found between these two grids. The wake peak is underestimated by both $\mathrm{G}_{1}$ and $\mathrm{G}_{2}$, but $\mathrm{G}_{3}$ reflects the experimental data well. For this reason, the mesh $\mathrm{G}_{3}$ was used for all further simulations. Although no grid convergence of this grid with regard to the total pressure loss in the wake can be observed, the grid resolution is high enough so that significant errors due to the grid can be excluded and the grid resolution is sufficient for a method comparison.

\section{Cascade with Incoming Wakes}

As reference total pressure for the calculation of the isentropic Mach number distribution and the total pressure losses in the wake, the total pressure between the bars and the cascade was chosen. In the experiment, however, only the total pressure upstream of the cascade was measured. Therefore, the total pressure upstream of the cascade was corrected by the total pressure losses of the row of bars from the simulation with meshed bars $\Delta p_{t, b a r}=121 \mathrm{~Pa}$, which is in good agreement with the experimental investigations of Clinckemaillie and Arts (2019).

In Fig. 6 left, the time averaged pressure distributions for the experiment, the simulations with meshed bars and W-DFSEM 

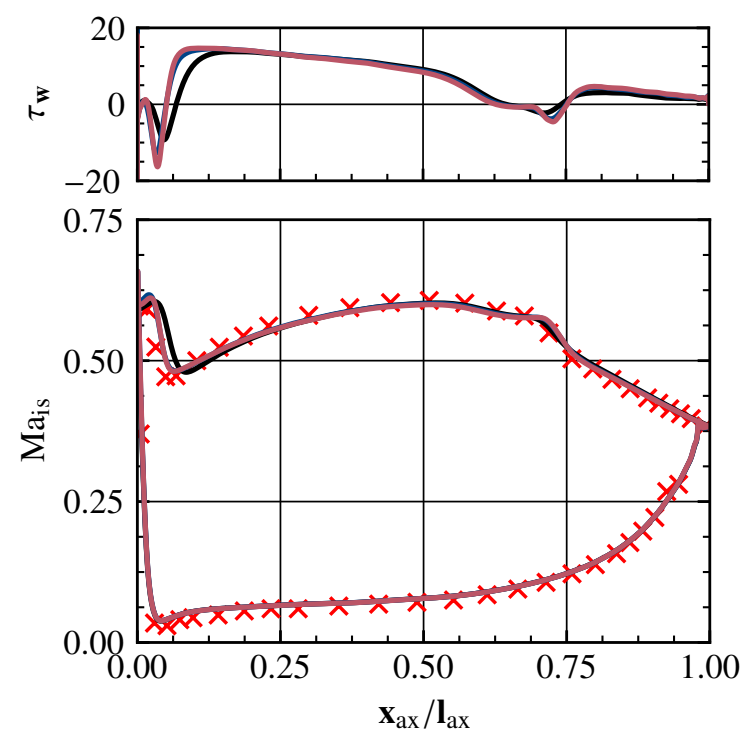

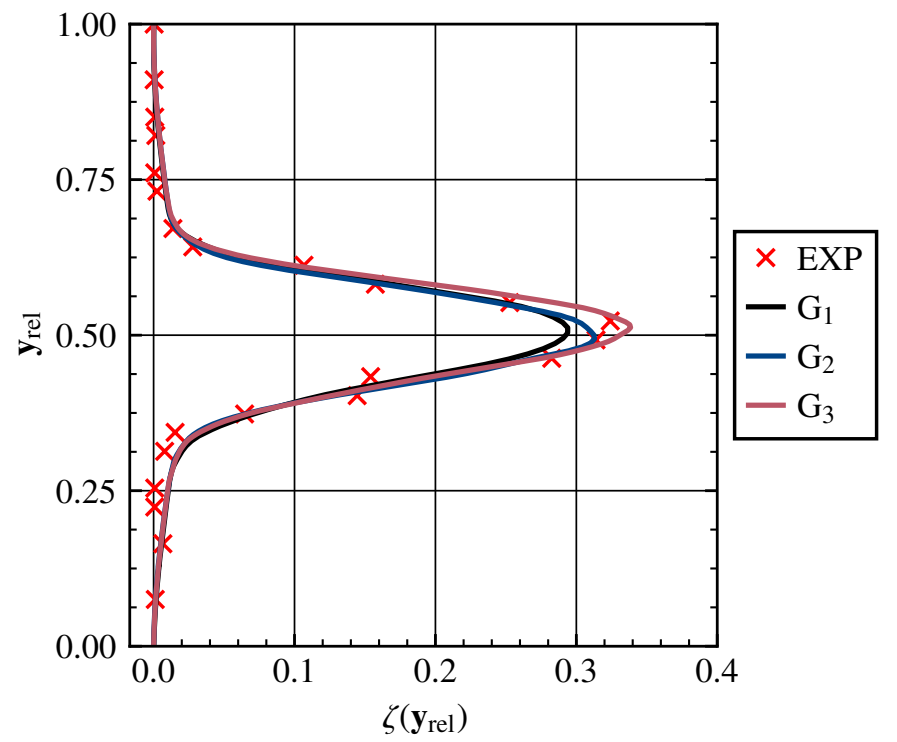

Figure 4 Wall shear stress at suction side (upper left), isentropic Mach number distribution at midspan (lower left) and total pressure losses in the wake at $x=1.47 l_{\text {ax }}$ (right) for the grid study of the cascade.

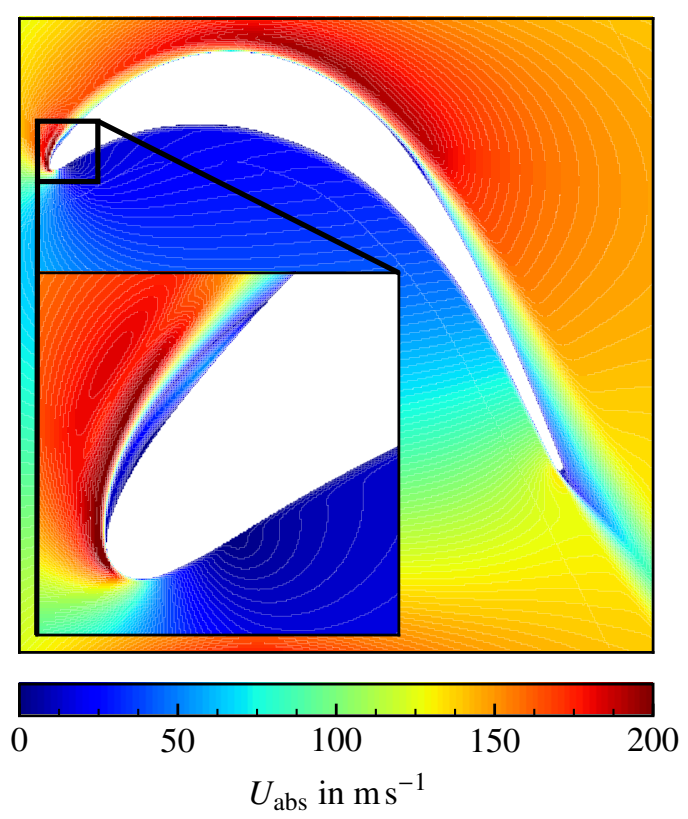

Figure 5 Velocity magnitude at midspan and detail view of the separation bubble at the leading edge

are compared. Both simulations can reproduce the pressure distribution of the experiment well. The deviations of both simulations from the experiment are in the same range as in the case without incoming wakes (see Fig. 4). A comparison of the total pressure loss in the wake of the cascade, graphically shown in Fig. 6 right, also shows a good agreement between the experiment and the simulations. The small differences between the simulations can probably be attributed to the different grid resolution. For the simulation with meshed bars the grid $\mathrm{G}_{2}$ was used. For the simulation with W-DFSEM the grid $\mathrm{G}_{3}$. The simulation with meshed bars was also initially started on the $\mathrm{G}_{3}$ mesh, but the necessary computational effort is unexpectedly high due to the poor parallelizability of the rotor-stator interface, which is why the grid resolution was reduced. However, the grid resolution of $\mathrm{G}_{2}$ is also high enough to reproduce the quantities of interest with sufficient accuracy (see Fig. 4).

In Fig. 7, the ensemble-averaged wall shear stresses of the simulations are additionally compared with the ensemble-averaged quasi wall shear stress from the experiment. In the experiment the wall shear stress is not measured directly, rather a quantity which is proportional to the wall shear stress. The the wall shear stresses in 7 were therefore scaled with a reference value $\tau_{\text {Ref }}$. This reference value is different for the experiment and simulations. For a detailed description of the determination of the quasi wall shear stress, please refer to (Stadtmüller, 2001). In addition to the wall shear stress, the separation and reattachment lines were drawn in white and the time offset between the earliest separation and the latest reattachment point $\Delta t / T$ is shown. Qualitatively, the simulations and the experiment are very similar. The results of the simulations are much more noisy due to the small number of averaged ensembles. A comparison of the axial positions shows a good agreement between experiment and W-DFSEM, whereas in the simulation with meshed bars, the separation and reattachment line are much further upstream, which is also due to the lower grid resolution. For the time offset $\Delta t / T$, the simulations deviate from the experimental value 0.32 , but are in the range of the experiment with 0.30 for the simulation with meshed bars and 0.27 for the simulation with W-DFSEM. 

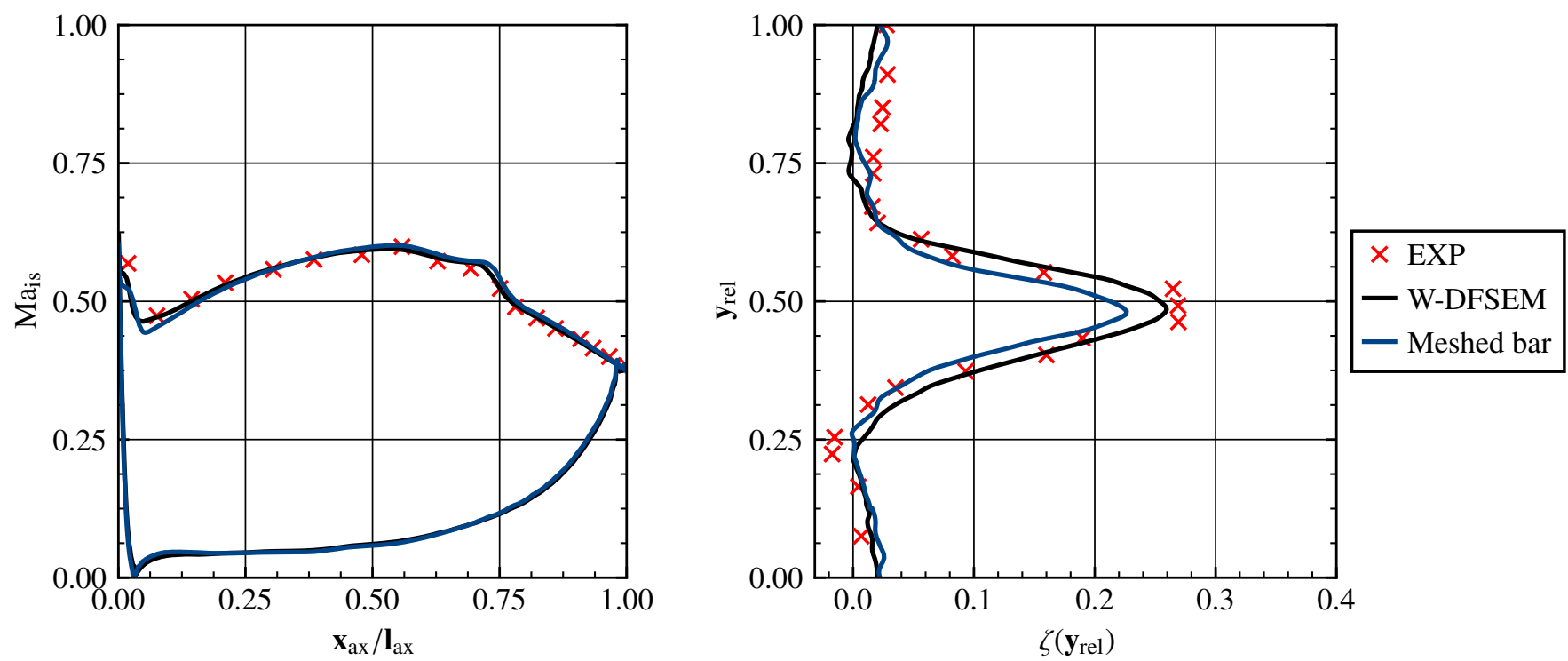

Figure 6 Isentropic Mach number distribution at midspan (left) and total pressure losses in the wake at $x=1.47 l_{\mathrm{ax}}$ (right) for the cascade testcase with incoming wakes
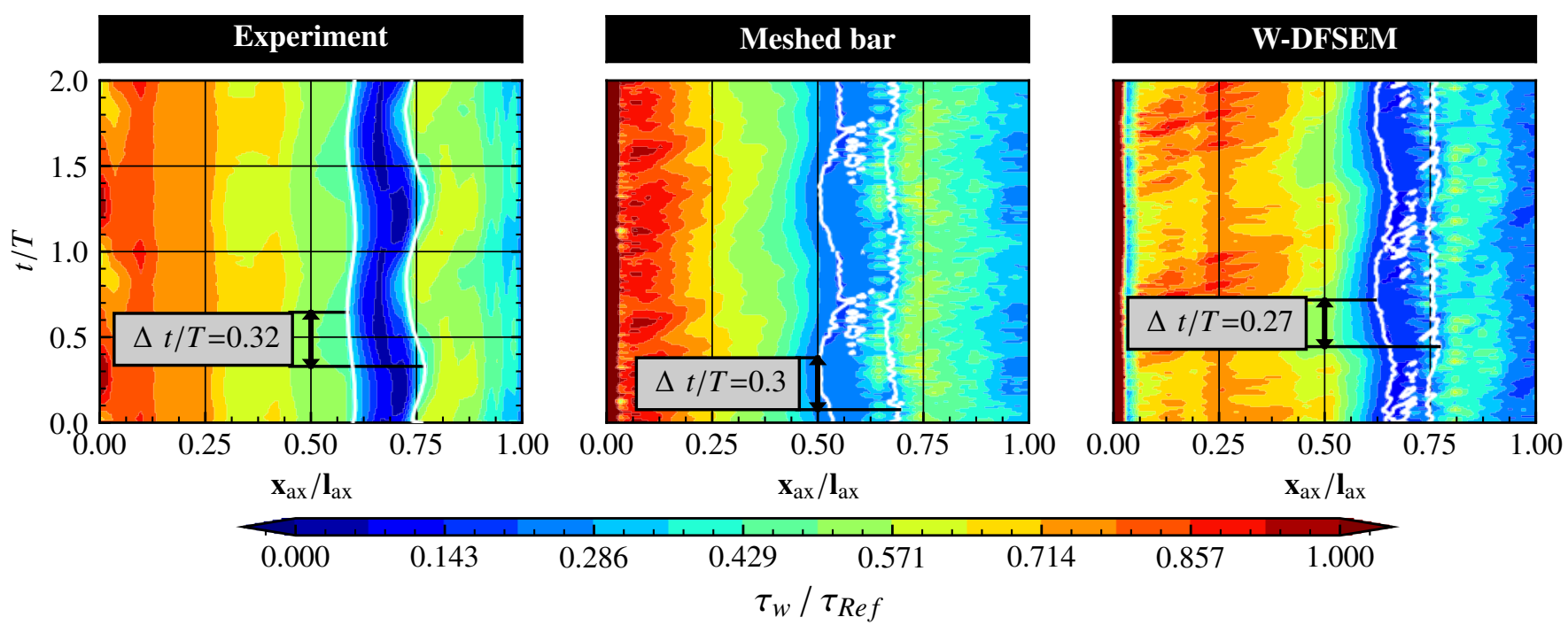

Figure 7 Comparison of the ensemble-averaged experimental quasi wall shear stress with the wall shear stress of the simulations with meshed bars and W-DFSEM for the suction side at midspan.

In summary, it can be said that the wakes generated by $\mathrm{W}$ DFSEM are not only largely identical to the experiment and simulation with directly meshed bars, but the complete modelling chain also correctly predicts the influence of these wakes on the downstream cascade. Thus, the second step of the validation concept is also fulfilled and the W-DFSEM is successfully validated.
Table 4 Comparison of the required computational ressources for the cascade with incoming wakes.

$$
\text { Ref W-DFSEM Meshed bar }
$$

Meshed bar without

RSI

$100 \% \quad 110 \% \quad 871 \% \quad 166 \%$

\section{Comparison of Computational Cost}

The simulations with W-DFSEM and with meshed bars are compared in Tab. 4 regarding the required computational 
resources. The values shown refer to the test case of the cascade with incoming wakes, but for the test case only with bar, the behavior is very similar. The simulation of the cascade without incoming wakes and the mesh resolution $\mathrm{G}_{3}$ served as a reference. The ratio of cells per processor was kept constant for all simulations and is about 15,000. The additional computational resources of W-DFSEM compared to the reference are $10 \%$. Although the additional number of cells between the simulation with meshed bars (23.4 million cells) and WDFSEM (20.7 million cells) is only $13 \%$, the additional needed computational resources for the simulations with meshed bars is more than $771 \%$ compared to the reference. This unexpectedly high computational effort can be explained by the poor parallelizability of the rotor-stator interface (RSI) and is also the reason for choosing a lower mesh resolution for the simulation with meshed bars. However, even without considering the rotor-stator interface, the additional computational effort of the simulations with meshed bars is significantly higher than with W-DFSEM (see Tab. 4). If, on the other hand, the influence of the wake of a profile and not of a bar were to be investigated, the number of cells would be about twice as high in the case of a meshed geometry upstream of the blade row and thus at least the additionally required computational resources. For these more realistic application cases, a significantly higher advantage of W-DFSEM with respect to the required computational resources can therefore be expected.

\section{CONCLUSIONS}

The aim of this study was the validation of the newly developed boundary condition Wakes and Compressible DivergenceFree Synthetic-Eddy Method (W-DFSEM) for the generation of realistic inflow conditions (including incoming wakes and free-stream turbulence) for scale-resolving simulations of turbomachinery cascades. The focus was especially on the synthetically generated incoming wakes. A validation concept was presented. The results of the simulations confirmed the following hypotheses...

1. The W-DFSEM allows the generation of realistic and physically plausible wakes.

2. The influence of these wakes on a downstream cascade is correctly reproduced throughout the entire modelling chain.

... and also showed that...

3. the direct generation of wakes by W-DFSEM at the inlet of a cascade simulation allows a significant reduction of computational resources compared to a simulation in which a wake's generating geometry is meshed and moved upstream of the cascade, and

4. these reduced computational costs, as well as the wide range of possible variations of the generated wakes of the W-DFSEM, allows the realization of extended parameter studies to investigate the influence of different wakes on a downstream cascade.
To summarize, the W-DFSEM has been successfully validated. To the author's knowledge, W-DFSEM can be considered as the first synthetic non-reflective boundary condition for scaleresolving simulations of compressible turbomachinery flows under various realistic inflow conditions. After successful validation, W-DFSEM can be used to investigate current blading and to gain new insights into turbulent wake-blade row interactions.

\section{NOMENCLATURE}

\section{Latin Symbols}

$\begin{array}{ll}b & \text { pitch } \\ N & \text { number of wakes, number of cells } \\ T & \text { bar period } \\ U_{\text {wake }} & \text { wake movement speed } \\ U_{F S} & \text { free-stream velocity } \\ U_{\text {abs }} & \text { velocity magnitude } \\ \Delta x^{+} & \text {tangential non-dimensional cell size } \\ \Delta y^{+} & \text {wall normal non-dimensional cell size } \\ y_{\text {rel }} & \text { relativ pitch } \\ y_{\text {wall }} & \text { wall normal distance first cell layer } \\ \Delta z^{+} & \text {spanwise non-dimensional cell size }\end{array}$

\section{Greek Symbols}

$\begin{array}{ll}\Delta_{i} & \text { average cell size far away from wall } \\ \omega & \text { wake width } \\ \sigma & \text { wake depth } \\ \tau_{\mathrm{w}} & \text { wall shear stress }\end{array}$

\section{Characteristic numbers}

$\mathrm{Re} \quad$ Reynolds number

Ma Mach number

$\begin{array}{ll}\text { Subscripts } & \\ \text { in, out } & \text { inlet, outlet } \\ \text { is } & \text { isentropic } \\ \text { ax } & \text { axial length }\end{array}$

\section{Acronyms}

C-DFSEM Compressible Divergence-Free Synthetic-Eddy Method

DFSEM Divergence-Free Synthetic-Eddy Method

EXP Experiment

I-DFSEM Incompressible Divergence-Free SyntheticEddy Method

LES Large-Eddy Simulation

RF Refinement factor

RSI Rotor-stator interface

SRS Scale-Resolving Simulations

URANS Unsteady Reynolds-averaged Navier-Stokes

WALE Wall adapting local eddy-viscosity

W-DFSEM Wakes and Compressible Divergence-Free Synthetic-Eddy Method 


\section{ACKNOWLEDGMENT}

We would like to thank the University of the Armed Forces in Munich for providing the cascade for this study and the authors acknowledge the North-German Supercomputing Alliance (HLRN) for providing HPC resources that have contributed to the research results reported in this paper.

\section{Appendix: Modelling equations of W-DFSEM}

The W-DFSEM only influences the velocity field at the inlet of the simulation. For reasons of simplification, firstorder temporal discretization is assumed in the following. The velocity at any point of the inlet patch $u_{i, n}$ is then given in Einstein notation by:

$$
u_{i, n}=\frac{u_{i, n-1}+k\left(U_{\text {Gauss }}+u_{i}^{\prime}\right)}{1+k}+c_{k} \frac{1+\alpha+k}{1+k} \frac{d u_{i}^{\prime}}{d t} .
$$

The factor $k$ is part of the property of wave permeability and is defined as follows:

$$
k=\frac{w d t}{l_{\infty}}
$$

The variable $w$ corresponds to the velocity of the structures that leave the domain through the patch considered:

$$
w=\max (\vec{u} \cdot \vec{n}+a ; 0),
$$

formed with the patch normal velocity $\vec{u} \cdot \vec{n}$ and the speed of sound $a$. Thus $w \neq 0$ applies to the structures leaving the domain. The degree of reflection of the boundary condition can be adjusted by the characteristic relaxation length $l_{\infty}$. The variable $\alpha$ represents a dimensionless propagation velocity related to the local cell width $\Delta x$ :

$$
\alpha=\frac{w d t}{\Delta x}
$$

For a detailed description of the wave permeability property, please refer to the original paper (Poinsot and Lelef, 1992).

The velocity deficit of the wake $U_{\text {Gauss }}$ is based on a Gauss function and is given by

$$
U_{\text {Gauss }}(t, y)=U_{F S} \cdot\left(1-\frac{1}{\sqrt{2 \pi \sigma^{2}}} \exp \left(-\frac{(f(t, y) \omega)^{2}}{2 \sigma^{2}}\right)\right)
$$

with an additional function

$$
\begin{array}{r}
f(t, y)=\left(\frac{\left[(y-h(t, y)+g(y)-\min (y)) \bmod \left(\frac{b}{N}\right)\right]}{\frac{b}{N}}\right. \\
-0.5) \cdot \frac{10.0}{N} .
\end{array}
$$

The movement of the wake in pitch direction is controlled using the function

$$
h(t, y)=U_{\text {wake }} t \bmod \left[\operatorname{sign}\left(U_{\text {wake }}\right) b\right] .
$$

The function

$$
\begin{gathered}
g(y)=\left[\frac{|y-(\min (y)+0.5 b)|}{0.5 b}-1\right] 0.5 b . \\
\operatorname{int}\left(\frac{y-(\min (y)+0.5 b)}{0.5 b}\right) .
\end{gathered}
$$

ensures that the values are always within one pitch. For a detailed derivation of the respective function or velocity deficit, please refer to (Zieße et al., 2019).

To reproduce the turbulent fluctuations in the wake, eddies are continuously seeded by the boundary condition and convected through the inlet patch. The discretization of the eddy fluctuations $u_{i}^{\prime}$ only occurs when the eddies pass the inlet patch and result from

$$
u_{\beta}^{\prime}(x)=\sqrt{\frac{1}{N}} \sum_{k=1}^{N} \sigma_{\beta}^{k}\left[1-\left(d^{k}\right)^{2}\right] \epsilon_{\beta j l} r_{j}^{k} \alpha_{l}^{k}
$$

with $N$ eddies, the Levi-Civita symbol $\epsilon$, length scale $\sigma_{\beta}^{k}$, intensity $\alpha_{m}^{k}$ of the $k$-th eddy and the overall distance from the patch cell to the eddy center $d^{k}$. The eddy intensity $\alpha_{m}^{k}$ follows directly from the prescribed Reynolds stress field $R$. To enable the specification of anisotropic turbulence, the eddies are additionally rotated from the principal axes system $(\mathrm{P})$ to the global coordinate system $(\mathrm{G})$ by using the Reynolds stress tensor:

$$
u_{i}^{\prime G}(x)=C_{1} R_{i m}^{P \rightarrow G} u_{m}^{\prime P} .
$$

$u_{m}^{\prime}$ and $u_{i}^{\prime G}$ are the velocity fluctuations along the principal axes and and in the global system respectively, $R_{i m}^{P \rightarrow G}$ the rotation transformation matrix and $C_{1}$ a normalization coefficient. For a more detailed description of the synthetic eddies please refer to (Poletto et al., 2013).

The movement of the turbulent field is realized by a repositioning and additional convection of the eddies in pitch direction. After the initial random positioning the eddies are repositioned in pitch direction by an offset

$$
\begin{array}{r}
\Delta y_{m}=t \cdot U_{\text {wake }} \bmod \left(\operatorname{sign}\left(U_{\text {wake }}\right) \cdot b\right) \\
+b \cdot\left(\operatorname{sign}\left(0.5 b-t \cdot U_{\text {wake }} \bmod \left(\operatorname{sign}\left(U_{\text {wake }}\right) \cdot b\right)\right)+1\right) / 2 .
\end{array}
$$

The additional convection in pitch direction results directly from the shift velocity of the wake $U_{\text {wake }}$ :

$$
\Delta y=\Delta t U_{\text {wake }} .
$$

\section{References}

[1] Müller, C., Herbst, F., Fiala, A., Zscherp, C., Kuegeler, E., and Seume, J. "Parameter Study for an Improved Prediction of Wake-Induced Transition in Low-Pressure Turbines". In: International Gas Turbine Conference (Nov. 2015). 
[2] Stadtmüller, P., Fottner, L., and Fiala, A. "Experimental and Numerical Investigation of Wake-Induced Transition on a Highly Loaded LP Turbine at Low Reynolds Numbers". In: Turbo Expo: Power for Land, Sea, and Air Volume 3: Heat Transfer; Electric Power; Industrial and Cogeneration (May 2000).

[3] Berrino, M., Simoni, D., Ubaldi, M., Zunino, P., and Bertini, F. "Off-Design Performance of a Highly Loaded LP Turbine Cascade Under Steady and Unsteady Incoming Flow Conditions". In: Turbo Expo: Power for Land, Sea, and Air Volume 2D: Turbomachinery (June 2014).

[4] Dhamankar, N., Blaisdell, G., and Lyrintzis, A. "An Overview of Turbulent Inflow Boundary Conditions for Large Eddy Simulations (Invited)". In: 22nd AIAA Computational Fluid Dynamics Conference (June 2015).

[5] Zieße, M., Müller-Schindewolffs, C., Seume, J., and Herbst, F. "Enhanced Synthetic-Eddy-Method for Generating Moving Incoming Wakes and Turbulence in ScaleResolving Simulations”. In: International Gas Turbine Conference (Nov. 2019).

[6] Stadtmüller, P. and Fottner, L. "A Test Case for the Numerical Investigation of Wake Passing Effects on a Highly Loaded LP Turbine Cascade Blade". In: Turbo Expo: Power for Land, Sea, and Air Volume 1: Aircraft Engine; Marine; Turbomachinery; Microturbines and Small Turbomachinery (June 2001).

[7] Hilgenfeld, L., Stadtmüller, P., and Fottner, L. "Experimental Investigation of Turbulence Influence of Wake Passing on the Boundary Layer Development of Highly Loaded Turbine Cascade Blades". In: Flow, Turbulence and Combustion 69 (Dec. 2002), pp. 229-247.

[8] Blaim, F. and Niehuis, R. "Unsteady Simulation of the LP Turbine Test Case T106D-EIZ Using a Transport Equation Based Transition Model". In: Deutscher Luftund Raumfahrtkongress 2012 (2013).

[9] Stadtmüller, P. "Investigation of wake-induced transition on the LP turbine cascade T106D-EIZ - TEST CASE DOCUMENTATION". In: DFG-Verbundprojekt Fo 136/11 - Periodisch Instationäre Strömungen in Turbomaschinen Version 1.1 (2001).

[10] Franck, N. and Ducros, F. "Subgrid-Scale Stress Modelling Based on the Square of the Velocity Gradient Tensor". In: Flow Turbulence and Combustion 62 (Sept. 1999), pp. 183-200.

[11] Jasak, H. "Dynamic Mesh Handling in OpenFOAM". In: 47th AIAA Aerospace Sciences Meeting including The New Horizons Forum and Aerospace Exposition (Jan. 2009).

[12] Ferziger, J. and Peric, M. Computational Methods for Fluid Dynamics. Springer Berlin Heidelberg, 2001. ISBN: 9783540420743.

[13] Poinsot, T. and Lelef, S. "Boundary conditions for direct simulations of compressible viscous flows". In: Journal of Computational Physics 101.1 (July 1992), pp. 104129.

[14] Jarrin, N., Prosser, R., Uribe, J., Benhamadouche, S., and Laurence, D. "Reconstruction of turbulent fluctuations for hybrid RANS/LES simulations using a SyntheticEddy Method". In: International Journal of Heat and Fluid Flow 30 (June 2009), pp. 435-442.

[15] Poletto, R., Craft, T., and Revell, A. "A New Divergence Free Synthetic Eddy Method for the Reproduction of Inlet Flow Conditions for LES”. In: Flow, Turbulence and Combustion 91 (Oct. 2013), pp. 1-21.

[16] Müller-Schindewolffs, C. and Herbst, F. “The Effect of Turbulent Scales on Low-Pressure Turbine Aerodynamics: Part A - An Optimized Turbulent BoundaryCondition". In: Turbo Expo: Power for Land, Sea, and Air Volume 2B: Turbomachinery (June 2018). V02BT41A004.

[17] Lakshminarayana, B. and Poncet, A. "A Method of Measuring Three-Dimensional Rotating Wakes Behind Turbomachinery Rotors". In: Journal of Fluids Engineering 96.2 (June 1974), pp. 87-91.

[18] Michelassi, V, Wissink, J. G., and Rodi, W. "Direct numerical simulation, large eddy simulation and unsteady Reynolds-averaged Navier-Stokes simulations of periodic unsteady flow in a low-pressure turbine cascade: A comparison". In: Proceedings of the Institution of Mechanical Engineers, Part A: Journal of Power and Energy 217.4 (2003), pp. 403-411.

[19] Michelassi, V., Chen, L.-W., Pichler, R., and Sandberg, R. D. "Compressible Direct Numerical Simulation of Low-Pressure Turbines-Part II: Effect of Inflow Disturbances". In: Journal of Turbomachinery 137.7 (July 2015).

[20] Koschichow, D., Fröhlich, J., Kirik, I., and Niehuis, R. "DNS of the Flow Near the Endwall in a Linear Low Pressure Turbine Cascade With Periodically Passing Wakes". In: Proceedings of the ASME Turbo Expo 2 (June 2014).

[21] Tennekes, E. P. o. A. E. H., Tennekes, H., Lumley, J. L., Lumley, J. L., and Technology, M. I. o. A First Course in Turbulence -. Cambridge: MIT Press, 1972.

[22] Clinckemaillie, J. and Arts, T. "Characterization of Periodic Incoming Wakes in a Low-Pressure Turbine Cascade Test Section by Means of a Fast-Response Single Sensor Virtual Three-Hole Probe". In: International Journal of Turbomachinery, Propulsion and Power 4.3 (Aug. 2019), p. 26. 